\title{
Evolving landscapes in multiple sclerosis research: adaptive designs and novel endpoints
}

\author{
Manolo E. Beelke ${ }^{1 *}$, Paola Antonini ${ }^{2}$ and Michael F. Murphy ${ }^{3}$
}

\begin{abstract}
The increasing amount of highly effective treatment options in relapsing forms of multiple sclerosis (MS) requires innovative clinical trial design strategies. These strategies may encompass the application of adaptive designs as well as the adoption of innovative primary outcome measurements. The offered advantages would include, among others, shorter study follow-up periods and reduction in the number of patients either on placebo or on non-suitable dosages of the small molecules or biological products under examination. Changing the primary endpoint during the study conduct additionally represents an option, when the primary endpoint originally is either a composite endpoint of Magnetic Resonance Imaging (MRI) and clinical variables or a unitary endpoint of clinical variables. The new outcome measurement of no-evidence-of-disease activity (NEDA) - the former disease activity free (DAF) status, might represent an attractive approach and NEDA may become a new standard for clinical trials in relapsing MS (RMS), particularly for pivotal Phase III trials, though also earlier phase trials and exploratory clinical research might benefit from this endpoint. Future studies in RMS could incorporate NEDA as a primary endpoint and utilize the adaptive design methodology in order to reduce the sample size and the duration of new therapeutic agents' clinical development.
\end{abstract}

Keywords: Relapsing multiple sclerosis, No evidence of disease activity (NEDA), Disease activity free (DAF), Adaptive design, Primary endpoint

\section{Table of contents}

\section{Abstract 3 \\ Introduction 4 [NEDA] status 9 \\ Conclusions 13 \\ Abbreviations 15 \\ Competing interests 15 \\ Author contributions 15 \\ Author information 15}

Adaptive study design methodology in the clinical development of products targeting multiple sclerosis 6 Learning, confirming or both? 8

An interim change of the primary dependent variable - use of the No-Evidence-of-Disease Activity
Acknowledgements 16

References 17

\section{Background}

Since the commercial availability of disease-modifying treatments (DMTs) for the treatment of relapsing multiple sclerosis (RMS), significant changes have occurred in the diagnosis and management of multiple sclerosis (MS), with a significant impact on the clinical research in this area [1]. The year 1993 saw the release of the first disease-modifying drug, Betaseron ${ }^{\circ}$ and this was accompanied by increased research and attention on the disease. Since this time, patients have developed an increasingly sophisticated and accurate understanding of MS [2]. Additionally, the increasing number of clinical trials has allowed investigators to observe changes in terms of characteristics and behaviors of clinical trial populations in RMS [3, 4]. In particular, patient populations in recent studies are characterized by a lower

\footnotetext{
Correspondence: manolo.beelke@wwctrials.com

${ }^{1}$ Medical \& Scientific Affairs, Worldwide Clinical Trials (WCT), Landsberger Str.

63A, 82110 Germering, Germany

Full list of author information is available at the end of the article
} 
clinical disease activity level at the time of entry into clinical trials in comparison to the past. These observations limit the utility of historical data for effect size estimations based upon either the number or time to the events or other aspects of disease progression as points of interest. The reasons for observations of improved placebo response across many indications are variable, but within RMS this shift might be related mainly to two factors:

1. The adoption of McDonald criteria that allows shortening significantly the timeframe from the status of a clinically isolated syndrome to the diagnosis of MS [5].

2. The current standard of care of starting treatment early, which makes nowadays treatment-naïve subjects available for clinical trials only when they are at a very early stage of the disease [6-17].

Given these changes in patient phenotype, two main challenges can be identified when designing a future trial in MS:

1. The classical clinical endpoints, recommended by European Medicines Agency's (EMA's) Guideline on clinical investigation of medicinal products for the treatment of Multiple Sclerosis [18], may not be sensitive enough to demonstrate additional efficacy with respect to the current standard treatments in head-to-head comparison studies. Even if statistically significant differences are demonstrated within primary study data sets it could be argued whether the detected difference would constitute any clinically meaningful improvement. Clinical trials with an add-on treatment design might face similar challenges.

2. The chance of detecting differences with respect to placebo in a population with low disease activity is reduced.

3. Any shift during the clinical development with a clinical trial conducted in Phase II on a study population with low disease activity towards a specific population with high disease activity, such as in a Phase III confirmatory trial, may be accompanied by incorrect assumptions regarding the nature of the dose-exposure-response relationships, erroneous power calculations for planned sample sizes, and differences in the hierarchy of primary and secondary measures. This may also incur potentially significant regulatory restrictions in the drug label as a consequence of changes in the patient population and in the nature of assessments occurring within the development program.
New design strategies are therefore required to handle this evolution in patient presentation and progression, given that trial methodologists historically have had limited choices for a Phase III trial. One standard option has been to increase the sample size and the number of subjects on placebo, introducing potential ethical issues [19]. Alternatively, use of a head-to-head comparative or add-on treatment study designs, both designs with active treatment groups, addresses the ethical issue of exposure to placebo, but adds further challenges due to the expected small difference in efficacy between two active treatments using traditional measures. From this original situation, creative solutions have been sought and employed which go beyond these two standard options and resolve some of their inherent shortcomings.

Among others, a strategy of replacement of the current standard primary endpoints with a new one could produce an increased level of sensitivity without the loss of specificity and reliability. In addition, especially in early exploratory studies, some advantageous options include:

- Enrichment maneuvers in selecting the patient population, ensuring high disease activity status, for example through the use of a run-in period during which monthly Magnetic Resonance Imaging (MRI) scans are performed.

- Use of an adaptive design in order to prospectively allow the change of a dependent variable or sample size based upon interim information during the study.

- Use of surrogate MRI markers as primary outcomes, or as outcomes that can be used for dose selection purposes antecedent to longer-term studies evaluating clinical outcomes.

For the purpose of this article, the potential benefits offered by adaptive study designs and new outcome measures will be presented in detail.

\section{Adaptive study design methodology in the clinical development of products targeting multiple sclerosis} Adaptive designs have received attention in the last few years by both the pharmaceutical/biotechnology industries and health authorities/regulatory agencies. Adaptive clinical design methodology, and the terminology to describe this methodology, has evolved over the years [20]. Interchangeable terms historically have included adaptive, sequential, flexible, self-designing, multi-stage, dynamic and response driven models [21]. Differences in terminology have contributed to inconsistent and confusing perspectives regarding benefits and potential disadvantages of these designs. 
For the pharmaceutical and biotechnology industries, adaptive designs represent a diverse methodology with the common purpose of improving efficacy in the clinical development of drugs, with additional advantages in enhancing efficiency by reducing the cost and time components associated with drug development. Generally aggregated under the umbrella of "learning phase" versus "confirmatory phase" investigations, a portfolio of different adaptive designs support different objectives, and range in maturity from decades old methodology (for example, group sequential designs) to more recent and relatively underutilized study designs such as the use of seamless Phase II/III studies [22]. Frequently, combinations of two or more approaches are encountered as shown in Table 1.

However, debate and discussion have followed the publication of recent Food and Drug Administration (FDA) guidance on adaptive designs [23]. While this guidance recognizes the urgent need for new drug development tools, and sets a standard template for adaptive designs, it also emphasizes potential risks in the introduction of bias through the use of an adaptive study design methodology. According to the FDA guidance, an adaptive design should possess three features:

1.) It is a prospectively planned modification of one or more specified element(s) of study design or hypotheses.

2.) The modification is performed in a pre-specified manner based on an interim analysis of data from subjects in the study, at one or more times during the trial.

3.) The interim analyses of accumulated study data are performed at pre-specified time points, in a fully blinded or unblinded manner, and can occur with or without formal statistical hypothesis testing.

Table 1 Learning phase versus confirmatory phase investigations Learning Phase Paradigms

- Adaptive dose-finding for toxicity (Bayesian or Frequentist)

- Adaptive dose finding for efficacy (Bayesian or Frequentist)

- Seamless phase I/lla

Confirmatory Phase Paradigms

- Group sequential

- Sample size re-estimation

- Adaptive randomization based on observed response

- Adaptive randomization based upon covariate

- Enrichment Designs

- Adaptive treatment-switching design

- Adaptive seamless phase II/II

Adaptive hypotheses

- Superiority to non-inferiority
As opposed to traditional fixed design trials, adaptive trials use accumulated data to modify some aspects of the ongoing trial, with the goal of preserving the validity and integrity of the trial. Frequently, adaptive design methodology requires a commensurate increases in study simulations, unique methods of trial management, and occasionally less than intuitive methods of analyses and interpretation. It is axiomatic that the complexity of an adaptive design must not exceed the available infrastructure to support its design and execution. Low complexity adaptive designs within an environment of well-established infrastructure and processes yield the best prospects for success.

\section{Learning, confirming or both?}

The utility of adaptive clinical trial methodology should be especially seen in the context of exploratory research and proof of concept trials, although there have been adaptive trials submitted as a component of marketing authorization [24]. In a drug development program, pure exploration is usually the theme in early phase studies, where the dose-exposure-response relationships are speculative, and the clinical phenotype of the intended patient population and the primary efficacy endpoint are under study. Adaptation for sample size estimation, the discontinuation of uninformative dosage groups, or enrichment of the patient sample represent the most common utilizations. Once preliminary data supports the Proof-of-Concept, then a large-scale, randomized, well-controlled and more traditional confirmatory trial may be pursued.

In the central nervous system (CNS) area there are examples reported in the literature of drug development programs that utilized exploratory adaptive designs prior to continuing the drug development program or launching registration trials. A prominent example of this is acute migraine trials [25]. More complex adaptive designs may include both patient adaptation and dose adaptation, incorporating the flexibility to re-estimate the sample size or the selected dose arm(s) [26].

An adaptive design may combine different phases of clinical trials; for example, the seamless adaptive Phase II/III design might be envisioned to combine Phase II and Phase III trials into one trial without a time gap between the two phases.

Beyond the use of group sequential designs, other adaptive design approaches within the neurological spectrum of disorders are less frequently encountered, although new entrants, particularly for adaptive randomization purposes, have been recently reported [27]. Adaptive designs within MS are limited to very few examples as reported in the literature. Most frequently, the sample size estimation will be adapted in relation to the baseline disease activity of a limited group of subjects 
already randomized at the time of the interim analysis for this purpose [28].

There are, however, some other examples on how the adaptive design methodology can be applied to clinical development in MS. One of these is the BOLD study, a Phase II study with an adaptive, dose-ranging design [29]. In this study, an adaptive design was chosen to characterize the dose-response curve of the study drug. In a first period of study ("Period 1"), three doses of study drug and placebo were tested for MRI-supported efficacy. Based on an interim analysis after 3 months of treatment, two additional active doses for a second period of the study ("Period 2") were selected, thus optimizing the overall determination of the dose-response curve using 5 data points from active treatment doses along with placebo. The doses were kept blinded. The use of this design allowed for the determination of the optimal dose for later Phase III studies. The choice of placebo as a treatment control was considered essential to obtain information on the specific versus non-specific effects of the active treatment, and provided the best way of evaluating the efficacy, safety and tolerability profiles of the study drug. The use of an adaptive design strategy contributed to a significant reduction in placebo exposure, both in terms of the number of patients and in regards to study duration, compared to conventional trial models.

The structural and analytic aspects of the study conducted by Selmaj et al. have resemblance to other studies within neurological conditions such as amyotrophic lateral sclerosis (ALS), in which a two-stage seamless design is utilized in order to incorporate information regarding dose-response into a subsequent phase of the investigation [30]. With appropriate modifications to the design structure and taking into consideration the endpoints and disease features, these data might be informative for future RMS studies.

\section{An interim change of the primary dependent variable - use of the No-Evidence-of-Disease Activity [NEDA] status}

The FDA guidance mentions the option of adaptation for endpoint selection based on interim estimates of treatment effect. Primary endpoint revision usually takes one of two forms:

(1) Replacement of the designated primary endpoint with an entirely new endpoint, or

(2) Modification of the primary endpoint by adding or removing data elements to the endpoint (e.g., the discrete event types included in a composite event endpoint).

Therefore, one of the possible endpoint scenarios uses a composite endpoint instead of a unitary primary endpoint.
The selection of composite endpoints has also been regarded as "a useful strategy" for registration of pharmaceuticals in the International Conference on Harmonisation (ICH) E9 guideline. It has long been recognized within other therapeutic areas that using a composite endpoint to enhance prospects for signal detection at a desired level of statistical significance is often accompanied by potential distortions in the nature and magnitude of treatment effects. This is primarily related to an asymmetry in the clinical importance of various parameters constituting the composite - i.e., frequent, less clinically important outcomes can drive the effect of therapy on any composite [31]. Therefore, the regulatory requirements for the use of composite endpoints have been specified by the EMA Committee for Proprietary Medicinal Products (CPMP) [32].

According to the CPMP document, the use of a composite endpoint in a clinical trial is usually justified if the following assumptions are respected:

- The individual components of the composite are clinically meaningful and of similar importance to the patient [33].

- The expected effects on each component are similar, based on biological plausibility [34]. Accordingly, regulatory guidelines also require the use of components for which it can be assumed that treatment will beneficially influence them in a similar way.

- The clinically most important components of composite endpoints should at least not be affected negatively (no worsening of the clinical condition as measured by the component).

As a consequence, regulatory authorities require that each component of a composite endpoint be analyzed separately [32] to assist in the interpretation of treatment effect, even if the composite is statistically significant.

In MS, the disease-activity-free (DAF) status or as more recently called, the no-evidence-of-disease activity (NEDA) status, has become a new treatment goal in daily clinical practice, and is used for decision making on treatment changes or dose adaptations for current treatments [35]. NEDA, a composite measure of disease activity, is currently defined only by 3 parameters:

- Absence of new or enlarging T2 legions or T1 gadolinium-enhancing lesions on MRI.

- Absence of sustained Expanded Disability Status Scale (EDSS) score progression.

- Absence of any clinical relapse.

The concept of NEDA however, in the combination of the three variables as listed above or with any additional 
parameter proposed (NEDA-4), is currently lacking any standardization in terms of frequency of assessments and time period of observation. It is only recommended that NEDA should be evaluated within a given time frame of at least 1 year. Under these circumstances, the NEDA status could be strongly influenced by the duration of the study, the disease activity present at baseline, and the frequency of preplanned assessments This might not only be particularly relevant for the MRI component, but also for the clinical components for which the observation of disease progression is directly in relationship to the duration of the observational period. Indeed, a newly published research aimed to establish a reasonable study duration, as for the predictive value of this parameter on the long-term disease activity, determined that with an observational period of 2 years, NEDA can predict disability at 7 years almost as well as at 5 years [36].

All 3 parameters defining NEDA status represent different aspects of the disease activity in MS, and considering them together as representation of the disease activity increase the sensitivity for detecting evidence of disease activity, as measurable nowadays. The choice of NEDA as new outcome measurement, as defined by a composite of the 3 parameters, increases statistical precision and efficiency, with beneficial effects on sample size and study duration. The use of NEDA thus builds upon a tradition of combining imaging and clinical parameters in RMS where such combinations are thought to provide more sensitive outcome measures [37].

However, composite endpoints are associated with both clinical and biostatistical encumbrances, as shown already [38] in other therapeutic areas, and emphasized by the CPMP document [32] as well. It is therefore reasonable to approach their construction in MS with comparable caution. Similarly, there are strong arguments in the recent literature against the use of NEDA as a primary outcome in clinical trials designated for regulatory approval purposes [35], even if it's recognized as an important goal for treating individual patients with relapsing disease or as a method for selecting the most appropriate therapy to move forward into confirmatory trials. In addition, the high variability of disease progression is neglected by this outcome measure. While Imitola [39] argues that the concept of NEDA might not be a realistic outcome for patients with MS, especially with increasing duration of disease, NEDA might demonstrate the most utility during the early stages of relapsing-remitting phase. Indeed, since none of the current available treatments for RMS represents a "cure" of the disease, they can only be called disease modifying treatments (DMTs). DMTs are able only to reduce the frequency and severity of relapses, extend the time intervals between relapses, and slow progression to permanent disability [40]. Thus, in the long-term perspective very few subjects will maintain the status of NEDA, as the disease will progress even under treatment with DMTs.

Another potential downside in the use of this outcome lies in the risk of the overall measure being driven by the event with the greatest level of activity, as opposed to all events equally, thus violating the symmetry principle required for composite endpoints. Indeed, the disease activity free status might represent a reflection of only MRI defined activity events in trials in RMS where the composite has been employed, without balancing between frequency of assessments and the likelihood for observed findings within the MRI component as compared to the clinical ones. This risk is discussed by Nixon et al. [41], who states how some analytical adjustment may be required to account for the dominance of one component measure when the overall composite endpoint is driven to a large extent by MRI outcomes, with minimal contribution from clinical measures. Such unbalance is further emphasized by the number of observational time points used, if the number of MRI time points is higher than those for clinical assessments. MRI assessments present a higher sensitivity than clinical assessments, and disease progression in terms of clinical assessments is less likely to occur than disease progression shown by MRI. Indeed, if NEDA is used as a primary endpoint in a Phase III confirmatory trial, there is a risk of simply reproducing the results of a Phase II (MRI-driven) study instead of producing novel clinical evidence of treatment efficacy, if MRI impact is not properly weighted, because relapses are rare events and also disease progression develops very slowly over time. Although the utility of NEDA status endpoint in early phase interventional research, as well as in observational studies, is easily recognizable, further discussion with health authorities is required to enable inclusion of this outcome measure in the guidelines on new entities' clinical development and registration (i.e. potentially pivotal) investigations in RMS. Due to its peculiar variability, it should be considered that the use of NEDA as a new primary endpoint from a methodological viewpoint may significantly affect sample size estimation, and it may also introduce a challenge in weighing each component in order to avoid unbalance and bias in estimation of comparative effects. Nevertheless, if properly used, NEDA would represent a powerful new primary endpoint even in pivotal Phase III trials, circumnavigating many of the current statistical challenges inherent in clinical-based endpoints.

\section{Conclusions}

Over the past 20 years, significant changes have occurred in the diagnosis and management of MS, with a significant impact on clinical research in this area. Additionally, the disease-related characteristics of patients presenting for 
evaluation in prototypical interventional studies has changed. The combination of novel DMTs along with a change in the clinical trials environment, mandate examination of different clinical trial methodologies. These include structural modifications in study design as well as the creation of novel pharmacodynamic and disease-related endpoints. The advent of new effective therapies in particular has made the use of placebo in trials lasting more than 6 months difficult to justify clinically, although methodological rigor precludes the sole use of active control comparative trials as a basis for deducing evidence of efficacy and safety.

Research in RMS is focusing on more sensitive clinical outcomes and study designs which can more efficiently evaluate the dose-exposure-response relationship of innovative therapies in early phase clinical trials, while simultaneously reducing the number of patients exposed to ineffective treatments in clinical programs. We have identified the recently proposed concept of "No-evidence-of disease-activity" (NEDA) status as a possible new parameter to be used in conjunction with adaptive trial designs to investigate the efficacy of new treatment strategies in RMS. Despite several limitations that the disease itself imposes for endpoint qualification and despite the prudent approach of health authorities towards the use of adaptive design for later phase investigations, the prospect of employing an adaptive design for endpoint selection based on interim estimate of treatment effect represents an attractive and plausible program option, even in pivotal Phase III trials. The use of NEDA as an endpoint deserves further evaluation for future exploratory studies in MS. Particular attention should be given to the methodological aspects of this endpoint, including the duration of the observational period and the weighing of each single component. Further methodological research and modeling are needed, as well as a full standardization of NEDA as an endpoint, before this can be universally accepted as a primary endpoint in future pivotal trials.

\section{Abbreviations}

CNS: central nervous system; CPMP: Committee for Proprietary Medicinal Products; DAF: disease-activity-free; DMTs: disease modifying treatments; EDSS: expanded disability status scale; EMA: European Medicines Agency; FDA: Food and Drug Administration; ICH: International Conference on Harmonisation; MRI: magnetic resonance imaging; MS: multiple sclerosis; NEDA: no-evidence-of-disease activity; RMS: relapsing multiple sclerosis.
\end{abstract}

\section{Competing interests}

All authors are paid employees at Worldwide Clinical Trials. The authors declare that they have no competing interests. The statements contained in this manuscript reflect the personal opinion of the authors and should not be construed as representing the opinions of Worldwide Clinical Trials.

\section{Authors' contributions}

MEB, PA and MFM all contributed equally to writing, editing and approving the final manuscript.

\section{Authors' information}

Dr. Beelke is board certified Clinical Neurophysiologist and Neurologist, and involved in the Clinical Development in Multiple Sclerosis for more than 12 years. He received his M.D. from the University of Genoa, Italy and a PhD in Sleep Medicine from the University of Bologna, Italy.

Dr Antonini received her M.D. from the University of Rome, Italy. She also has a PhD in Clinical Pharmacology. Her medical training experience includes an internship in oncology surgery at the Cancer Research Institute of Rome, Italy. In the current position at WCT Dr Antonini is responsible for scientific services delivery.

Dr. Murphy is a board-certified psychiatrist and pharmacologist, Research \& Development Editor for American Health \& Drug Benefits ${ }^{\mathrm{TM}}$ and a lecturer within the Master's Program in Clinical and Translational Investigation of the Harvard Clinical and Translational Science Center.

\section{Acknowledgements}

Editorial assistance and medical writing support in the preparation of this manuscript was provided by Andrew Kuhlman at Worldwide Clinical Trials.

\section{Author details}

${ }^{1}$ Medical \& Scientific Affairs, Worldwide Clinical Trials (WCT), Landsberger Str. 63A, 82110 Germering, Germany. ${ }^{2}$ Worldwide Clinical Trials (WCT), Via Giorgio Ribotta 21, 00144 Rome, Italy. ${ }^{3}$ Worldwide Clinical Trials (WCT), 1000

Continental Drive, Suite 290, King of Prussia, PA 19406, USA.

Received: 22 July 2015 Accepted: 17 February 2016

Published online: 01 April 2016

\section{References}

1. Cutter $G$, Kappos L. Clinical trials in multiple sclerosis. Handb Clin Neurol. 2014;122:445-53. doi:10.1016/B978-0-444-52001-2.00019-4.

2. Kraft GH, McMullen KA, AM B. The changing face of multiple sclerosis: Differences in gender and symtoms over a 27-year period. Int J MS Care. 2009;11(S2):4

3. Uitdehaag BM, Barkhof F, Coyle PK, Gardner JD, Jeffery DR, Mikol DD. The changing face of multiple sclerosis clinical trial populations. Curr Med Res Opin. 2011;27(8):1529-37. doi:10.1185/03007995.2011.591370.

4. Nicholas R, Straube S, Schmidli H, Schneider S, Friede T. Trends in annualized relapse rates in relapsing-remitting multiple sclerosis and consequences for clinical trial design. Mult Scler. 2011;17(10):1211-7. doi:10.1177/1352458511406309.

5. Polman CH, Reingold SC, Edan G, Filippi M, Hartung HP, Kappos L, et al. Diagnostic criteria for multiple sclerosis: 2005 revisions to the "McDonald Criteria". Ann Neurol. 2005:58(6):840-6. doi:10.1002/ana.20703.

6. Goodin DS, Bates D. Treatment of early multiple sclerosis: the value of treatment initiation after a first clinical episode. Mult Scler. 2009;15(10): $1175-82$.

7. Confavreux C, Vukusic S, Adeleine P. Early clinical predictors and progression of irreversible disability in multiple sclerosis: an amnesic process. Brain. 2003; 126(4):770-82.

8. Flachenecker P, Rieckmann P. Early intervention in multiple sclerosis: better outcomes for patients and society? Drugs. 2003;63(15):1525-33.

9. Frohman EM, Havrdova E, Lublin F, et al. Most patients with multiple sclerosis or a clinically isolated demyelinating syndrome should be treated at the time of diagnosis. Arch Neurol. 2006;63(4):614-9.

10. Sormani MP, Bonzano L, Roccatagliata L, Mancardi GL, Uccelli A, Bruzzi P. Surrogate endpoints for EDSS worsening in multiple sclerosis: a metaanalytic approach. Neurology. 2010;75(4):302-9.

11. Pakdaman $\mathrm{H}$, Sahraian MA, Fallah A, et al. Effect of early interferon beta-1a therapy on conversion to multiple sclerosis in Iranian patients with a first demyelinating event. Acta Neurol Scand. 2007;115(6):429-31.

12. Hughes R, Francis G. PRISMS-4: long-term efficacy of interferon- $\beta$-1a in relapsing MS. Neurology. 2001;56(12):1628-36.

13. Kappos L, Traboulsee A, Constantinescu C, et al. Long-term subcutaneous interferon beta-1a therapy in patients with relapsing-remitting MS. Neurology. 2006;67(6):944-53.

14. Garcea O, Villa A, Cáceres F, et al. Early treatment of multiple sclerosis: a Latin American experts meeting. Mult Scler. 2009;15(3):S1-12.

15. O'Connor P, Devonshire V. The use of disease-modifying agents in multiple sclerosis-by the Canadian network of MS clinics. Can J Neurol Sci. 2008; 35(2):127-32. 
16. Karussis D, Biermann LD, Bohlega S, et al. A recommended treatment algorithm in relapsing multiple sclerosis: report of an international consensus meeting. Eur J Neurol. 2006;13(1):61-71.

17. National Multiple Sclerosis Society USA (NMSS), "Disease management consensus statement [online]," 2015, http://www.nationalmssociety.org/ getmedia/5ca284d3-fc7c-4ba5-b005-ab537d495c3c/DMT_Consensus_MS_ Coalition_color. Accessed 9 July 2015 and 14 March 2016

18. Guideline on clinical investigation of medicinal products for the treatment of Multiple Sclerosis, Rev 2. 26 March, 2015. http://www.ema.europa.eu/ docs/en_GB/document_library/Scientific_guideline/2015/03/WC500185161. pdf. Accessed 9 July, 2015.

19. Polman $\mathrm{CH}$, Reingold SC, Barkhof F, Calabresi PA, Clanet M, Cohen JA, et al. Ethics of placebo-controlled clinical trials in multiple sclerosis: a reassessment. Neurology. 2008;70(13 Pt 2):1134-40. doi:10.1212/01.wnl. 0000306410.84794.4d.

20. Temple R. FDA perspective on trials with interim efficacy evaluations. Stat Med. 2006;25(19):3245-9. doi:10.1002/sim.2631. discussion 326-47.

21. Dragalin V. Adaptive designs: terminology and classification. Drug Inf J. 2006:40:425-35.

22. Kairalla JA, Coffey CS, Thomann MA, Muller KE. Adaptive trial designs: a review of barriers and opportunities. Trials. 2012;13:145. doi:10.1186/1745-6215-13-145.

23. U.S. Food and Drug Administration. Draft guidance for industry: adaptive design clinical trials for drugs and biologics. February 2010. http://www.fda. gov/downloads/\%20DrugsGuidanceComplianceRegulatorylnformation/ Guidances/UCM201790.pdf. Accessed 14 July 2015.

24. Elsasser A, Regnstrom J, Vetter T, Koenig F, Hemmings RJ, Greco M, et al. Adaptive clinical trial designs for European marketing authorization: a survey of scientific advice letters from the European Medicines Agency. Trials. 2014;15:383. doi:10.1186/1745-6215-15-383.

25. Ho TW, Mannix LK, Fan X, Assaid C, Furtek C, Jones CJ, et al. Randomized controlled trial of an oral CGRP receptor antagonist, MK-0974, in acute treatment of migraine. Neurology. 2008;70(16):1304-12. doi:10.1212/01.WNL. 0000286940.29755 .61$.

26. Cui L, Hung HM, Wang SJ. Modification of sample size in group sequential clinical trials. Biometrics. 1999;55(3):853-7.

27. Lenz RA, Pritchett YL, Berry SM, Llano DA, Han S, Berry DA, et al. Adaptive, dose-finding phase 2 trial evaluating the safety and efficacy of ABT-089 in mild to moderate Alzheimer disease. Alzheimer Dis Assoc Disord. 2015. doi:10.1097/WAD.0000000000000093.

28. Schneider $\mathrm{S}$, Schmidli $H$, Friede $T$. Blinded sample size re-estimation for recurrent event data with time trends. Stat Med. 2013;32(30):5448-57. doi: 10.1002/sim.5977.

29. Selmaj K, Li DK, Hartung HP, Hemmer B, Kappos L, Freedman MS, et al. Siponimod for patients with relapsing-remitting multiple sclerosis (BOLD): an adaptive, dose-ranging, randomised, phase 2 study. Lancet Neurol. 2013; 12(8):756-67. doi:10.1016/S1474-4422(13)70102-9.

30. Levy G, Kaufmann P, Buchsbaum R, Montes J, Barsdorf A, Arbing R, et al. A two-stage design for a phase II clinical trial of coenzyme Q10 in ALS. Neurology. 2006;66(5):660-3. doi:10.1212/01.wnl.0000201182.60750.66.

31. Ferreira-Gonzalez I, Busse JW, Heels-Ansdell D, Montori VM, AkI EA, Bryant DM, et al. Problems with use of composite end points in cardiovascular trials: systematic review of randomised controlled trials. BMJ. 2007;334(7597): 786. doi:10.1136/bmj.39136.682083.AE.

32. Committee for Proprietary Medicinal Products (CPMP). Points to consider on multiplicity issues in clinical trials. 19 September 2002. http://www.ema. europa.eu/docs/en_GB/document_library/Scientific_guideline/2009/09/ WC500003640.pdf. Accessed 14 July 2015.

33. Freemantle N, Calvert M, Wood J, Eastaugh J, Griffin C. Composite outcomes in randomized trials: greater precision but with greater uncertainty? JAMA. 2003;289(19):2554-9. doi:10.1001/jama.289.19.2554.

34. Montori VM, Busse JW, Permanyer-Miralda G, Ferreira I, Guyatt GH. How should clinicians interpret results reflecting the effect of an intervention on composite endpoints: should I dump this lump? ACP J Club. 2005;143(3):A8.

35. Giovannoni G, Turner B, Gnanapavan S, Offiah C, Schmierer K, Marta M. Is it time to target No evident disease activity (NEDA) in multiple sclerosis? Mult Scler Relat Disord. 2015;4(4):329-33.

36. Rotstein DL, Healy BC, Malik MT, Chitnis T, Weiner HL. Evaluation of no evidence of disease activity in a 7-year longitudinal multiple sclerosis cohort. JAMA Neurol. 2015;72(2):152-8. doi:10.1001/jamaneurol.2014.3537.
37. Cohen JA, Reingold SC, Polman CH, Wolinsky JS. Disability outcome measures in multiple sclerosis clinical trials: current status and future prospects. Lancet Neurol. 2012;11(5):467-76. doi:10.1016/S1474-4422(12)70059-5.

38. Cordoba G, Schwartz L, Woloshin S, Bae H, Gotzsche PC. Definition, reporting, and interpretation of composite outcomes in clinical trials: systematic review. BMJ. 2010;341:c3920. doi:10.1136/bmj.c3920.

39. Imitola J, Racke MK. Is no evidence of disease activity a realistic goal for patients with multiple sclerosis? JAMA Neurol. 2015;72(2):145-7. doi:10.1001/jamaneurol.2014.3860.

40. Derwenskus J. Current disease-modifying treatment of multiple sclerosis. Mt Sinai J Med N Y. 2011;78(2):161-75. doi:10.1002/msj.20239.

41. Nixon R, Bergvall N, Tomic D, Sfikas N, Cutter G, Giovannoni G. No evidence of disease activity: indirect comparisons of oral therapies for the treatment of relapsing-remitting multiple sclerosis. Adv Ther. 2014;31(11):1134-54. doi:10.1007/s12325-014-0167-z.

\section{Submit your next manuscript to BioMed Central and we will help you at every step:}

- We accept pre-submission inquiries

- Our selector tool helps you to find the most relevant journal

- We provide round the clock customer support

- Convenient online submission

- Thorough peer review

- Inclusion in PubMed and all major indexing services

- Maximum visibility for your research

Submit your manuscript at www.biomedcentral.com/submit
) Biomed Central 